\title{
The relevance of being active
}

\author{
Since the field's inception, the study of active galactic nuclei has been central to extragalactic astronomy. The \\ plurality of ways in which these objects can be observed and their numerous links to other fields of astronomy \\ maintain their continued relevance.
}

\begin{abstract}
$t$ has been fifty years since the seminal paper by Donald Lynden-Bell 'Galactic nuclei as collapsed old quasars' - it was published in Nature in August 1969 - put forward the idea of supermassive black holes being the engine behind the enormous energy output of quasi-stellar objects, or quasars. Lynden-Bell's paper came just six years after Maarten Schmidt's realization that these extremely luminous quasars were at cosmological distances. These two papers combined, together with a series of observations during the 60 s and 70 s, mainly in the radio and in the ultraviolet and optical, gave rise to the concept of active galactic nuclei (AGNs) and kickstarted a whole new field of research in extragalactic astronomy.
\end{abstract}

AGNs, and quasars more specifically which were the first AGNs to be scrutinized due to their extraordinary luminosities were a fascinating new beast for astronomers as, prior to the realization of their true nature, they presented a number of puzzles. Their energy output aside, these objects were observed to vary within, at the time, unphysical timescales. Today we know that at the highest energies AGNs show variability on timescales of minutes or less. Causality arguments require that the emitting regions should be minuscule. But this was not the end of wonders. Radio observations of some AGNs revealed matter moving at apparent superluminal speeds. Special relativity similarly would then require intrinsic speeds very close to the speed of light at small angles from our line of sight.

Despite their vast distances, AGNs are some of the brightest objects in the sky. In ultraviolet and optical wavelengths they can easily outshine their host galaxies, despite their emission originating from a region the size of our Solar System. As it turns out, a supermassive black hole, surrounded by a geometrically thin accretion disk threaded by strong magnetic fields, can explain most of the phenomenology of these objects. Add an obscuring dust shroud at the edge of the accretion disk, clouds of ionized gas scattered close to the black hole and all the way out beyond the obscuring screen, and in some cases collimated streams of radio-emitting magnetized plasma, and you create the complex picture of what we believe today are the building blocks of an AGN. Another 25 years passed before Megan Urry and Paolo Padovani put forward the 'Unified schemes for radio-loud active galactic nuclei', where all these elements were brought together and combined with the idea that the angle at which we view these objects changes their multi-wavelength properties.

Since then, our knowledge of AGNs has been significantly enriched and in some cases fundamentally revised. For example, it is now accepted that what was then known as the dust 'torus' is rather a conglomeration of clouds that might have a more complicated geometry than previously thought (for example, see our 2017 Review Article by Ramos Almeida and Ricci). Similarly, there is now ample evidence that the ionized gas component is much more complex in morphology and kinematics than we believed before (see the Letter by Chelouche et al. in this issue). From the accretion disk out to the outer regions of the photoionized gas, we now know that winds play a key role in transporting energy and momentum from the inner regions outward, potentially even affecting the host galaxy of the AGN (see the Letter by He et al. in this issue and our March 2018 focus issue on AGN outflows and feedback).

Perhaps most interestingly and despite early evidence to the contrary, we now believe that AGN activity is highly temperamental. While the full lifecycle of an AGN can be up to tens to hundreds of millions of years (based, for example, on the extent of their radio jets), the actual duty cycle of each activity episode might be far shorter than that (of the order of tens to hundreds of thousands of years, sometimes even shorter). This temporal volatility is probably due to chaotic accretion onto the supermassive black hole. One aspect of this situation is reflected in the extreme variability of AGN emission across the electromagnetic spectrum. Another aspect, as exemplified in the last decade by tidal disruption events, is the fading or reignition of AGNs. While the nomenclature in the field is still largely non-unified, these systems are often dubbed changing-look or extreme variability AGNs. These flaring or fading AGNs (or in some cases the brightening of a quiescent supermassive black hole) can provide valuable insights into the accretion and photoionization physics of these objects (see the Letter by Trakhtenbrot et al. in this issue).

Exactly due to their variability, AGNs were among the first subjects of timedomain and transient astronomy. As can be seen from this issue of Nature Astronomy, the temporal behaviour of AGNs is very much a focus of the community. With numerous new time-domain astronomy facilities/programmes currently in their commissioning phase or being planned for the near future - including Pan-STARRS, the Large Synoptic Survey Telescope, the SkyMapper, the Zwicky Transient Facility and the Catalina Real-Time Transient Survey, just to focus on optical wavelengths - the interest in the temporal properties of AGNs will not subside any time soon.

Beyond the study of AGNs themselves, interesting as it might be, accreting supermassive black holes also offer us a window to other exciting science. From using AGNs to study the physics of the supermassive black holes at their centres (see our January Insight) and test general relativity (see the 2018 Letter by Mizuno et al.), to studying the intergalactic medium in the early Universe (see the 2018 Letter by Cooke and Fumagalli), understanding neutrinos (see the Letter by Gao et al. in our November 2018 issue) and constraining cosmological parameters and the nature of dark energy (see the Letter by Risaliti and Lusso in this issue), AGNs have long presented themselves as versatile tools of relevance to most areas of Galactic and extragalactic astronomy.

Published online: 7 March 2019 https://doi.org/10.1038/s41550-019-0730-2 